\title{
The Hemodynamic Study on the Effects of Entry Tear and Coverage in Aortic Dissection
}

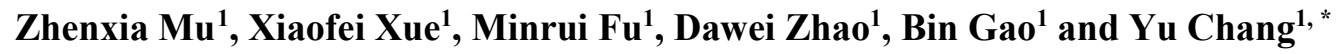

\begin{abstract}
In this work, the hemodynamic effects of the type-A aortic dissection in different entry and covering entry tear positions were mainly studied. It provides a new method or idea in the field of the aortic dissection hemodynamics, and it is of profound significance to provide basic theoretical research on the development of aortic dissection in the aspect of clinical judgment. Two type-A aortic dissection models with different entry tear positions (Model 1: The entry tear was located at the entrance of the ascending aorta, Model 2: The entry tear was located at the starting position of the descending aorta) were reconstructed according to the computed tomography (CT) images of the patients. In our study, the thoracic aortic endovascular repair was simulated by covering the entry tear (Model 3). To clarify the hemodynamic effects of entry tear and coverage, the comparative study on the true lumen (TL) and false lumen (FL) blood flow patterns of three models were carried out numerically. The velocity vector, flow ratio, pressure, time-averaged wall shear stress (TAWSS) and relative residence time (RRT) were calculated to evaluate the hemodynamic changes. The results of this work indicated that (I) the velocity of entry tear at the aorta entrance was higher; (II) The helical development of the TL and FL might be related to the helical nature of aortic arch; (III) The blood flow which passing the FL of Model 1, Model 2 and Model 3 in one cardiac cycle were approximately $26.63 \%, 13.39 \%$ and $1 \%$, respectively; (IV) The difference in intima wall pressure of the TL and FL were showed a strong pulsation; (V) The TAWSS distribution in TL and FL were completely different (the TAWSS in TL intima $>8 \mathrm{~Pa}$, the TAWSS in FL intima $<4 \mathrm{~Pa}$ ). In brief, the aortic morphology and location of the entry tear were found to have a significant effect on the hemodynamics of the aortic dissection. In addition, the CFD method is used to obtain multi-dimensional hemodynamic information such as velocity field, pressure, TAWSS and RRT, which can help clinicians better understand the development of type-A aortic dissection and provide a theoretical basis for clinical treatment.
\end{abstract}

Keywords: Computational fluid dynamics (CFD), aortic dissection, hemodynamic, TEVAR.

\section{Introduction}

Nowadays, cardiovascular disease has gradually been the number one killer of human health. Stanford type-A aortic dissection is a relatively common cardiovascular disease

\footnotetext{
${ }^{1}$ School of Life Science and BioEngineering, Beijing University of Technology, Beijing, 100124, China.

${ }^{*}$ Corresponding Author: Yu Chang. Email: changyu@bjut.edu.cn.
} 
with potential hazards [Hagan, Nienaber, Isselbacher et al. (2000)]. The disease is caused by a partial tearing of the aortic intima. Blood enters the intima-media from the aortic rupture, causing the dissection to tear and expand along the long axis of the aorta. Therefore, the initial single lumen of the aorta is divided into TL and FL [Golledge and Eagle (2008)]. The disease mortality rate is $1 \%-2 \%$ in 48 hours and $57 \%-89 \%$ in 2 weeks [Hebballi and Swanevelder (2009); Kallenbach, Oelze and Salcher (2004)]. Thoracic endovascular aortic repair (TEVAR) is one of the most effective methods for the treatment of type-A aortic dissection. At present, it has been widely used in the clinic by covering entry tear to achieve FL thrombosis and reduce pressure [Hanna, Andersen, Ganapathi et al. (2014)]. However, the effect of entry tears location on type-A aortic dissection and the prognosis of TEVAR intervention is unclear.

The development of computer technology is changing rapidly. Computational fluid dynamics (CFD) has been successfully applied to the hemodynamic study of cerebral aneurysms, abdominal aortic aneurysms and aortic dissection [Cebral, Castro, Burgess et al. (2005); Karmonik, Bismuth, Shah et al. (2011)]. The CFD can be used to provide information about blood flow patterns and pressure, which is a reliable tool for explaining disease development and evaluating the treatment effect. For the ordinary study of aortic dissection, some computational models are usually based on an artificially designed geometry. However, the patient-specific models based on medical imaging reconstruction can provide more accurate and convincing information [Alimohammadi, Sherwood, Karimpour et al. (2015)].

Researchers believe that aortic dissection may be caused by the abnormal aortic media structure and hemodynamics [Macura, Corl, Fishman et al. (2003)]. Shiyue et al. [Shi, Zhu, Chang et al. (2016)] found that the area of entry tear was larger than the re-entry tear, resulting in a more easily ruptured distal descending aorta and FL. Thomas et al. [Tsai, Schlicht, Khanafer et al. (2008)] studied the effect of B-type intima tear on the FL pressure and found that the diastolic FL pressure increased by $13.9 \%$ in the case of entry tear $(3.2 \mathrm{~mm})$ and re-entry tear thrombosis. They revealed that the increase of FL pressure led to the extension and rupture of the FL. Wan Naim et al. [Wan Ab Naim, Ganesan, Sun et al. (2018)] studied the differences in the velocity vector and wall shear stress distribution in 5 patients after TEVAR. They found that the wall shear stress of the aorta was lower and the FL was more prone to thrombosis in the cases of the entry tear position away from re-entry tear.

In this paper, three models (Model 1, Model 2, Model 3) were reconstructed to investigate the effects of different entry tear positions and coverage of the entry tear on flow behaviors including the velocity vector, distribution of wall pressure, time-averaged wall shear stress (TAWSS) and relative residence time (RRT). The results were extracted for evaluating the hemodynamic changes in TL and FL of the three models.

\section{Material and methods}

The method used in this study has been reported by the laboratory team [Shi, Zhu, Chang et al. (2016)]. Here, a detailed explanation will be given. 


\subsection{Aortic dissection model generation}

To evaluate the hemodynamic in the TL and FL, three geometric models were reconstructed based on a series of computed tomography angiography (CTA) images using the commercial 3D reconstruction software MIMICS (Materialise, Belgium). CTA images (in-plane resolution of 512 by 512 pixels with a pixel size of $0.625 \mathrm{~mm}$ and slice thickness of $1 \mathrm{~mm}$, total 600 images) were obtained from volunteers and approved by the institutional review board. Then the models were smoothed using Geomagic (Geomagic, USA) to improve the accuracy of the finite element analysis results. The aortic models were cut into thin layers of $1.2 \mathrm{~mm}$ as an anatomical section from the middle of the ascending aorta to the end of the thoracic aorta, along the aorta axis. The tear size and position of the three models were modified in the same way by computer-aided design (CAD) tools (SolidWorks 2015, SolidWorks, France):

Model 1: As shown, there were one entrance and one outlet tear, in which, the entry tear was located at the entrance of the ascending aorta (size: $8 \mathrm{~mm}^{2}$ ) and the re-entry tear was located at the end of the thoracic aorta (size: $16 \mathrm{~mm}^{2}$ );

Model 2: The entry tear was located at the starting position of the descending aorta (size: $8 \mathrm{~mm}^{2}$ ) and the re-entry tear was located at the end of the thoracic aorta (size: $16 \mathrm{~mm}^{2}$ ). There were one entrance and one outlet tear (reverse tear type-A aortic dissection);

Model 3: In the TEVAR process, the entry tear was covered (size: $0 \mathrm{~mm}^{2}$ ). The re-entry tear was located at the end of the thoracic aorta (size: $16 \mathrm{~mm}^{2}$ ), with only one outlet tear.

The main parts of the models included the ascending aorta, descending aorta, brachiocephalic artery, left common carotid artery, left subclavian artery, entry tear, and re-entry tear. The specific sizes and locations were shown in Fig. 1 and Tab. 1.
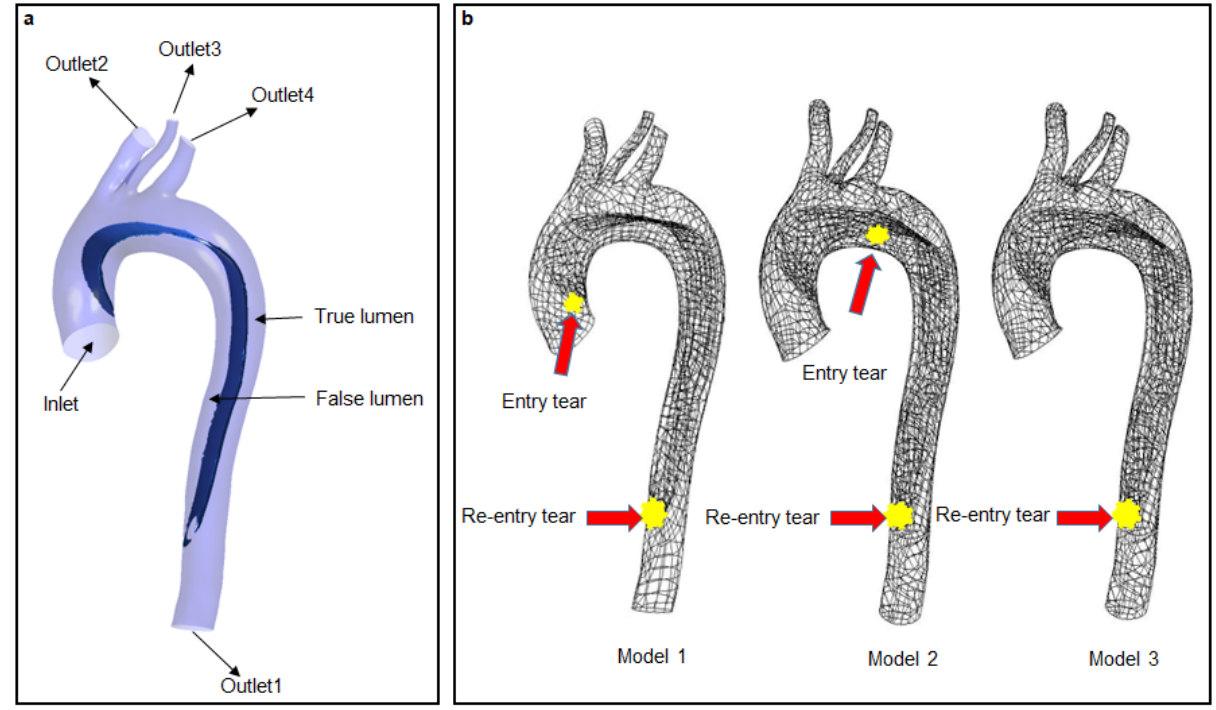

Figure 1: a: Patient-specific artery dissection geometry. b: The location of entry tears and re-entry tear in each model: entry tear is $8 \mathrm{~mm}^{2}$ and re-entry tear is $16 \mathrm{~mm}^{2}$ 
Table 1: Name, function and size of blood vessels

\begin{tabular}{cccc}
\hline Location & Name & Diameter $(\mathrm{mm})$ & Function \\
\hline Inlet & Ascending aorta & 31.14 & Inlet \\
Outlet1 & Descending aorta & 22.32 & Outlet \\
Outlet2 & Brachiocephalic artery & 11.95 & Outlet \\
Outlet3 & Left common carotid artery & 3.64 & Outlet \\
Outlet4 & Left subclavian artery & 10.16 & Outlet \\
\hline
\end{tabular}

\subsection{Meshing generation}

In this study, semi-automatic adaptive technology HyperMesh10.0 (Altair HyperWorks, Troy, MI, USA) mesh generator was used to generate high-quality tetrahedral 3D mesh. To obtain the optimal number of grids in this numerical computation, a grid independent analysis was conducted. The maximum WSS and pressure between grids were compared. Then the differences between the variables were calculated. According to the previous studies, the number of elements could be acceptable if the relative error was less than 5\%. In this study, 1.45 million elements were regarded as sufficient, and the element size was set to $1 \mathrm{~mm}$. Finally, the different inlet, outlet, TL and FL were defined by the mesh generator (Fig. 2). The numbers of elements and nodes in each model were shown in Tab. 2.

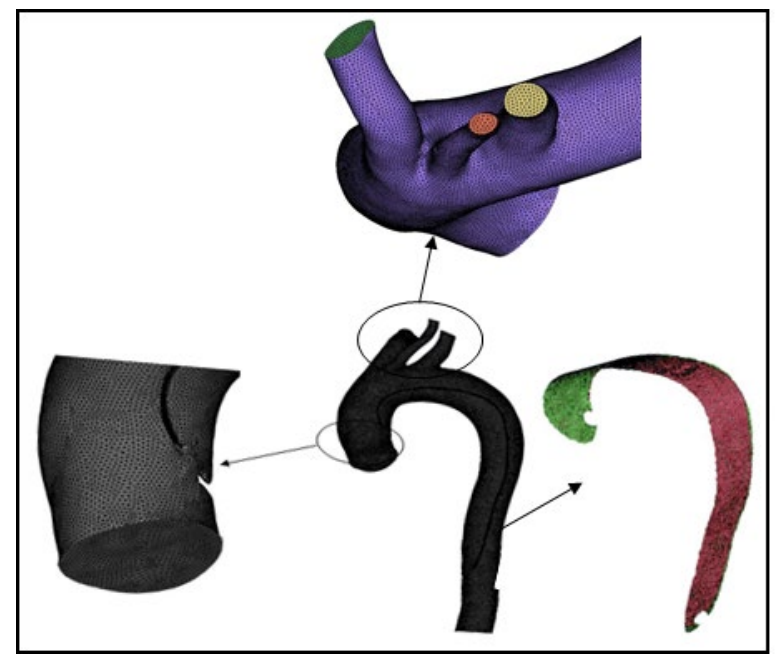

Figure 2: The mesh result of aortic dissection

Table 2: The numbers of elements and nodes in each model

\begin{tabular}{cccc}
\hline & Model 1 & Model 2 & Model 3 \\
\hline Element number & 1447445 & 1453245 & 1445623 \\
Node number & 252478 & 253012 & 252240 \\
\hline
\end{tabular}




\subsection{Numerical approaches}

The computation of aortic blood flow is based on the 3-dimensional incompressible Navier-Stokes Eqs. (1) and (2) [Remke (1981); Kabinejadian, Chua, Ghista et al. (2010)]:

$\rho \frac{\partial \mathbf{u}}{\partial t}+\rho(\mathbf{u} \cdot \nabla) \cdot \mathbf{u}=-\nabla p+\mu_{t} \nabla^{2} \mathbf{u}$

$\nabla \cdot \mathbf{u}=0$

where $\rho$ is the density of the fluid, $t$ is the time, $\mathbf{u}$ is the velocity vector, $p$ is the pressure, and $\mu_{t}$ is the viscosity of the blood turbulence. Navier-Stokes equations were solved in ANSYS / Fluent (Fluent 15.0, ANSYS, Inc., USA), utilizing a finite volumebased pressure correction algorithm and a second-order up winding scheme for the convective derivatives.

\subsection{Boundary conditions}

Due to the lack of patient-specific pressure information, the time-dependent pulsatile waveform of pressure boundary conditions at the aortic inlet was consistent with Rapezzis's work (Fig. 3(a)) [Rapezzi, Longhi, Graziosi et al. (2008)]. Time-dependent pulsatile waveform of velocity at the descending aorta outlet and the time-dependent pulsatile waveform of pressure at the brachiocephalic artery, left common carotid artery, and left subclavian artery were obtained from the work of Olufsen et al. [Olufsen, Peskin, Kim et al. (2000)] (Figs. 3(b) and Fig. 3(c)). In this study, the boundary conditions were consistent with previous researches.

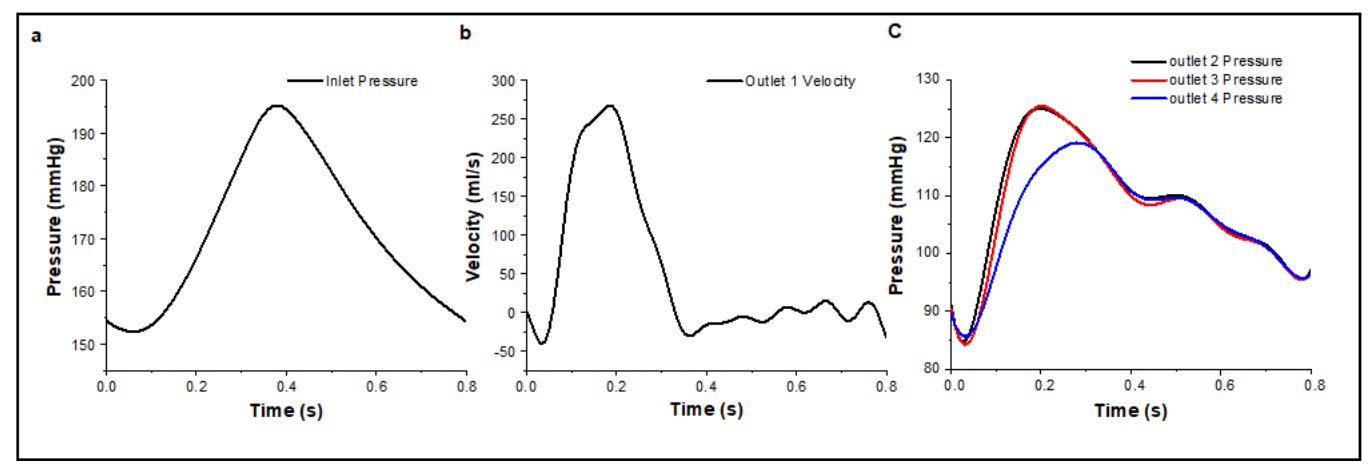

Figure 3: The boundary conditions in this study. (a) the blood pressure condition of the inlet; (b) the blood velocity condition of outlet 1; (c) the blood pressure condition of outlet 2 , outlet 3 and outlet 4

\subsection{Calculation settings}

In this study, the blood was assumed to be the homogeneous and incompressible Newtonian fluid [Aenis, Stancampiano, Wakhloo et al. (1997)]. The blood density and viscosity were set to $1050 \mathrm{~kg} / \mathrm{m}^{3}$ and $0.0035 \mathrm{~Pa} \cdot \mathrm{s}$, respectively [Zhang and Friedman (2013); Gijsen, van de Vosse and Janssen (1999); Angouras, Sokolis, Dosios et al. 
(2000)]. The maximum Reynolds number was 3208 in all models. The average Reynolds number calculated from the average velocity and average hydraulic diameter of the shrinkage peak was 944 . Based on the average hydraulic diameter, the Womersley number $(\alpha)$ was 23.9. Therefore, the blood was assumed to be laminar [Shi, Zhu, Chang et al. (2016); Chen, Müller-Eschner, Kotelis et al. (2013); Morris, Delassus, Walsh et al. (2004)]. The wall surface of the blood vessel was set to a non-sliding state. The cardiac cycle was set to $0.8 \mathrm{~s}$ and the calculation step was set to $0.01 \mathrm{~s}$. To obtain stable results, all models were simulated with four cardiac cycles to minimize the effects of initial flow conditions [Plonek, Zak, Rylski et al. (2018); Bonfanti, Balabani, Alimohammadi et al. (2018)]. The convergence precision was set to $10^{-5}$.

\subsection{Hemodynamic analysis}

The time-averaged wall shear stress (TAWSS), oscillatory shear index (OSI) and relative residence time (RRT) were calculated to illustrate the hemodynamic effects of entry tear and coverage on the aortic blood flow pattern.

TAWSS is used to describe the dynamic friction between a viscous fluid and a solid wall, which is caused by the lateral movement of a viscous fluid during a cardiac cycle. Where TAWSS is calculated as Eq. (3) [Ku (1997)]:

TAWSS $=\frac{1}{T} \int_{0}^{T}\left|\tau_{W}\right| d t$

where $\tau_{W}$ denotes the wall shear stress and $T$ is a cardiac cycle.

Higher OSI value is decisive factor leading to changes in intracellular membrane function and changes in cellular structural stress. The OSI is defined as Eq. (4) [Zhao, Liu, Bai et al. (2012)]:

$$
\mathrm{OSI}=0.5 \times\left(1-\frac{\left|\int_{0}^{T} \tau_{W} d t\right|}{\int_{0}^{T}\left|\tau_{W}\right| d t}\right)
$$

RRT is the time required for a particle to flow through a region. The RRT is defined as Eq. (5) [Himburg, Grzybowski, Hazel et al. (2004)]:

$$
\text { RRT }=1 /\{(1-2 \mathrm{OSI}) \mathrm{TAWSS}\}
$$

\section{Results}

To evaluate the differences of hemodynamic in TL and FL between the three models, the blood flow pattern in the aorta, flow ratios of TL and FL, wall pressure distribution, pressure d-value of the TL and FL intima, distribution of TAWSS and RRT were shown from Figs. 4-9.

\subsection{The distribution of blood flow pattern in the aorta dissection}

Fig. 4 shows the flow pattern distribution in the TL and FL at the velocity peak point of the three models. It has been observed that due to the tear in of the ascending and 
descending aortas, blood enters into the FL through the entry tear then enters back to the TL through the re-entry tear. At the velocity peak point, the blood flow velocity in the TL was always higher than that in the FL. The blood flow velocity was highest at the ascending aorta $(1.8 \mathrm{~m} / \mathrm{s})$ and the helical feature was also higher at the aortic arch. As shown, the blood flow direction in the descending aorta was parallel to the boundaries, making the flow more organized. Compared with Model 2 and Model 3 (the three figures in the second row), the FL of the Model 1 presented a more helical pattern in the ascending aorta and aortic arch. As shown in Fig. 4(a), the FL flow pattern of Model 1 was more complex. There was virtually no blood flow in the FL of Model 3 (Fig. 4(c)).

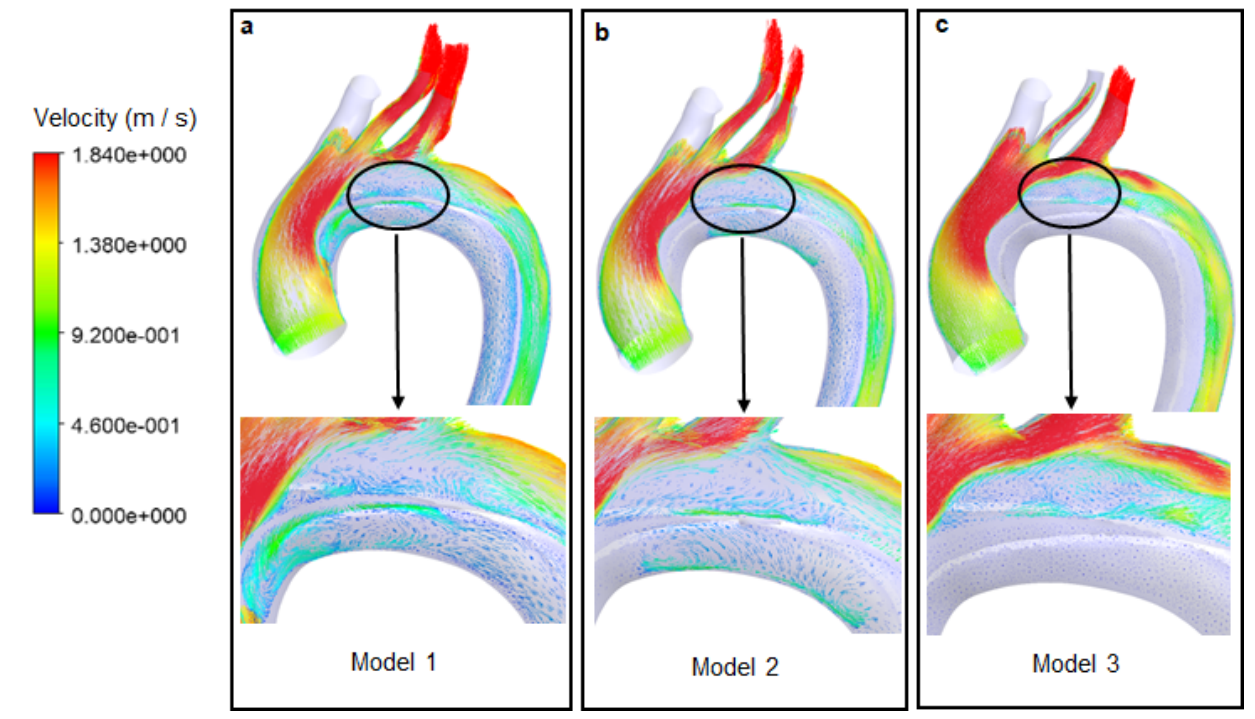

Figure 4: The flow pattern distribution in the TL and FL of the three models at the velocity peak point. The three figures in the second row are the flow patterns of the three models in the aortic arch

The most interesting area in aortic dissection research is the area of blood flow near the entry tear and re-entry tear. Therefore, the velocity magnitude slices were displayed in Fig. 5(a) (S 1, S 2 and S 3) to illustrate the hemodynamic changes near the entry tear and reentry tear at the velocity peak point. Fig. 5(b) shows the velocity vectors of the three models near the entry tear and the re-entry tear at the velocity peak point. As shown in Fig. 5(b) (Model 1 and Model 2), the blood flow velocity was faster through the entry tear and slower after entering the FL, resulting in obvious turbulence compared to the velocity vectors of S 1 and S 2. The flow field in the aortic arch was more complicated, especially when the velocity of blood flow was low. The phenomenon of blood flow vortex was more obvious. The blood flow velocity of the Model 1 at the entry tear $(1.82 \mathrm{~m} / \mathrm{s})$ was higher than that of Model $2(0.91 \mathrm{~m} / \mathrm{s})$. For Model 1 and Model 2, the blood flow velocity at the re-entry tear was higher than that of the surrounding area (the blood flow at the re-entry tear of model 1 was more obvious). For Model 3, the flow pattern became smoother and more 
regular at the re-entry tear. The flow pattern of each model was different, which was closely related to the morphological differences of the models.

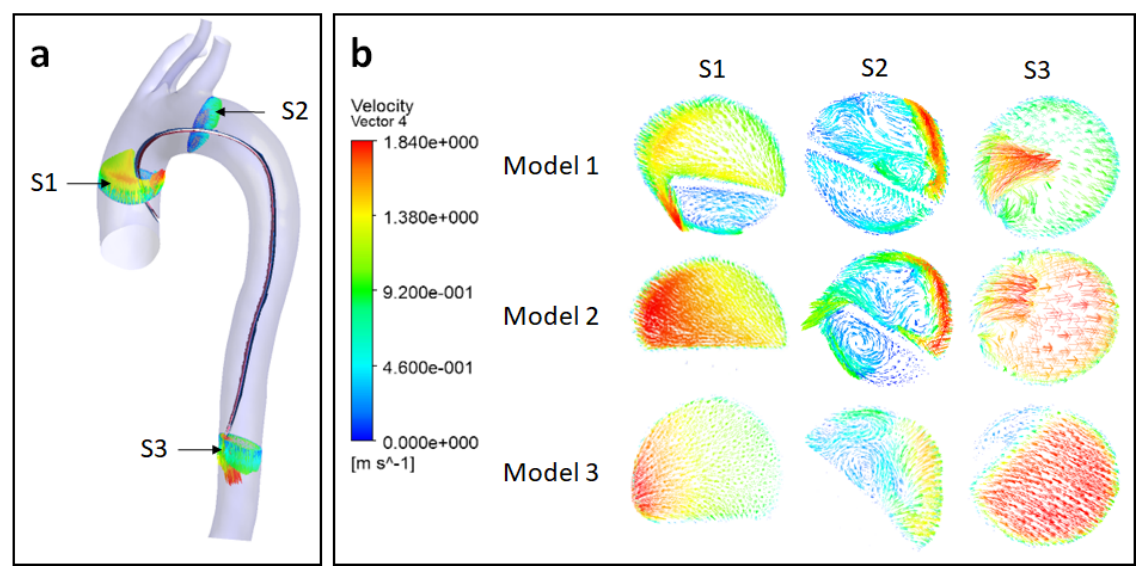

Figure 5: The velocity of three models at the velocity peak point. a: $\mathrm{S} 1$ is the entry tear of Model 1; S2 is the entry tear of Model 2; S3 is the re-entry tear of Model 1, Model 2 and Model 3. b: the velocity vectors of three models at special sections (S1, S2, S3)

Tab. 3 shows the flow ratio in the TL and FL in one cardiac cycle. The flow ratio in the FL of Model 1 was 26.63\%, and the flow ratio in the FL of Model 2 was $12.39 \%$. After covered the entry tear, the flow ratio in the FL of Model 3 was $1 \%$.

Table 3: The flow ratio in the TL and FL of three models in one cardiac cycle

\begin{tabular}{cccc}
\hline Flow ratio (\%) & Model 1 & Model 2 & Model 3 \\
\hline TL & $73.37 \%$ & $87.61 \%$ & $99 \%$ \\
FL & $26.63 \%$ & $13.39 \%$ & $1 \%$ \\
\hline
\end{tabular}

\subsection{The distribution of pressure in the aorta}

Fig. 6 shows the pressure distribution in the TL and FL at the velocity peak point. Fig. 6a shows the section of the pressure position and Fig. 6(b) shows the pressure of the three models. As shown in Fig. 6(b), it could be found that the lumen pressure of the three models gradually decreased from ascending aorta to descending aorta. More importantly, the pressure at the entrance of the aorta, brachiocephalic artery and left common carotid artery was relatively high (the black circle in Fig. 6(b)). Compared with Model 2 and Model 3, the FL pressure of Model 1 was higher (the average FL pressure of Model 1 was $1932 \mathrm{~Pa}$, the average FL pressure of Model 2 was $1898 \mathrm{~Pa}$, and the average FL pressure of Model 3 was $1858 \mathrm{~Pa}$ ). And for Model 1, the FL pressure of the descending aorta was higher than TL. In this study, the distribution of pressure is similar to previous studies [Shi, Zhu, Chang et al. (2016); Tsai, Schlicht, Khanafer et al. (2008); Wan Ab Naim, Ganesan, Sun et al. (2018); Remke (1981)]. 

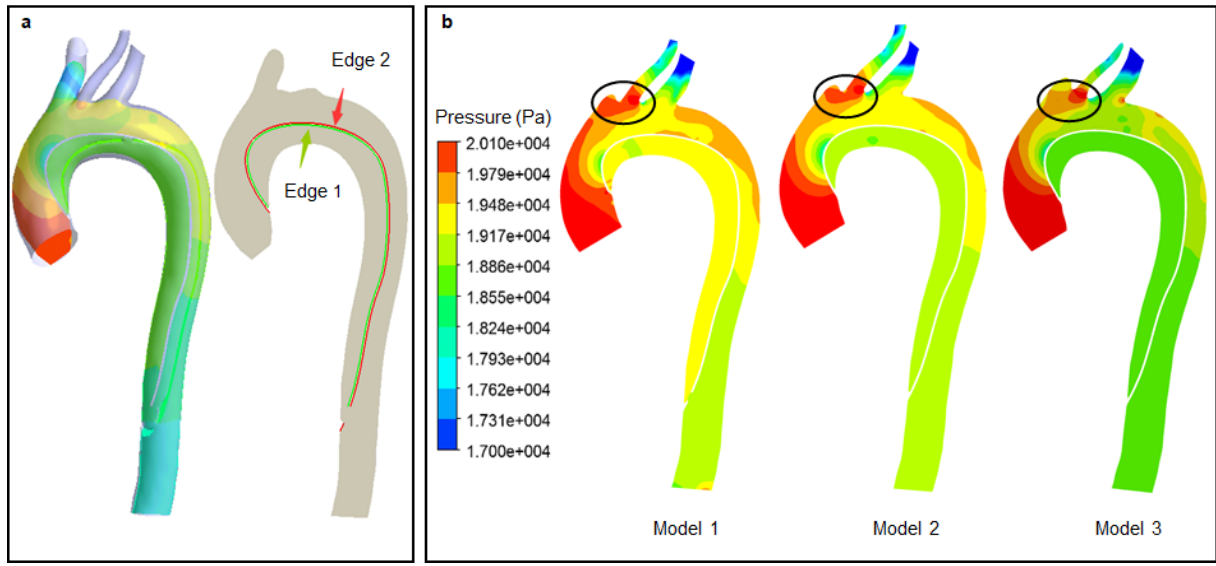

Figure 6: The pressure of three models at the velocity peak point. a: the section of the pressure position. $b$ : the pressure of three models

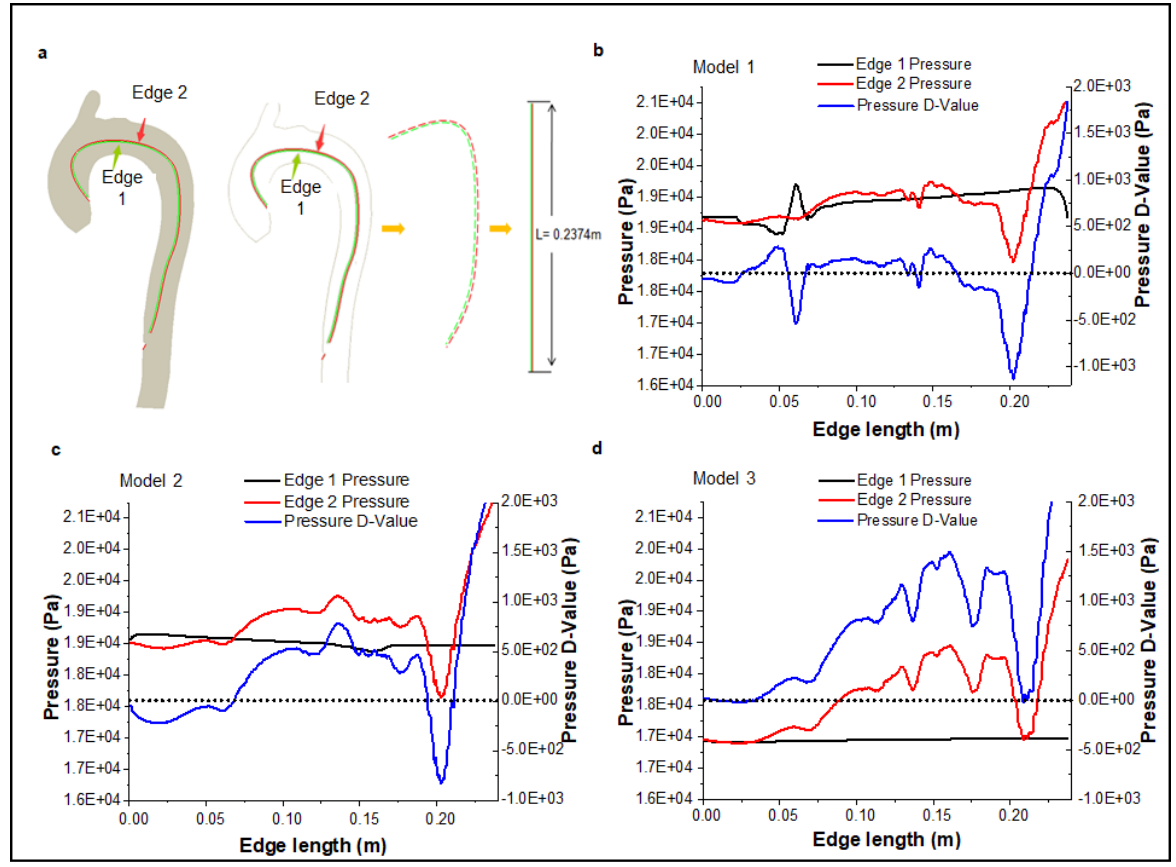

Figure 7: The pressure d-value of three models at the velocity peak point. a: Edge 1 is the intersection of the pressure surface and FL intima; Edge 2 is the intersection of the pressure surface and TL intima. b, c, d: the intima pressure and intima pressure d-value of Model 1, Model 2 and Model 3. The left ordinate axis is utilized to show the pressure of the TL and FL intima. The right ordinate axis is utilized to show the pressure d-value of the TL and FL intima

Fig. 7 shows the differential pressure of three models in the dissection intima wall at the velocity peak point. Fig. 7(a) shows the intersection of the dissection intima wall and the 
pressure section. Fig. 7(b) shows the differential pressure in the TL and FL (the pressure in the TL intima minus the FL intima). In this study, the edge length of the TL and FL intima was $0.2374 \mathrm{~m}$.

For Model 1, the pressure d-value in the TL and FL intima showed a strong pulsation from the ascending aorta to the descending aorta and the pressure in the FL intima was higher than that TL (the highest pressure d-value was 1128.5 Pa, Fig. 7(a)). Compared with Model 1, the pressure d-value of Model 2 intimal was relatively stable. The pressure in the TL intima at the entry tear was generally higher than FL, but opposite at the reentry tear (the highest pressure d-value was 823.4 Pa, Fig. 7). After covering the entry tear, the pressure in the FL intimal was reduced and the pressure in the TL intimal was higher than FL in one cardiac cycle (Fig. 7(c)). According to the position of pressure difference peak, it could be found that the tear position had a significant effect on the pressure distribution of TL and FL.

\subsection{The distribution of TAWSS in the aorta dissection}

WSS cannot be directly measured by the clinical experiments but can be given in CFD. TAWSS is calculated by averaging WSS in one cardiac cycle, which is a better representative of WSS. Fig. 8 shows the TAWSS in the aorta dissection intima of three models. Figs. 8(a) and 8(b) illustrate the distribution of TAWSS in FL and TL of Model 1. Figs. 8(c) and 8(d) show the distribution of TAWSS in FL and TL of Model 2. Figs. 8(e) and 8(f) illustrate the distribution of TAWSS in FL and TL of Model 3. The results showed that the TAWSS in the TL intima of three models was generally higher than that in FL (the TAWSS in TL intima $>8 \mathrm{~Pa}$, the TAWSS in FL intima $<4 \mathrm{~Pa}$ ). And the highest part of TAWSS in the TL intima was located at the ascending aorta and aortic ligament, where the flow pattern was disturbed and the velocity was very fast. The lower TAWSS area could be found in the FL intima, and the distribution of tear edge was unique in the descending aorta re-entry tear (Model 1>Model 2>Model 3).

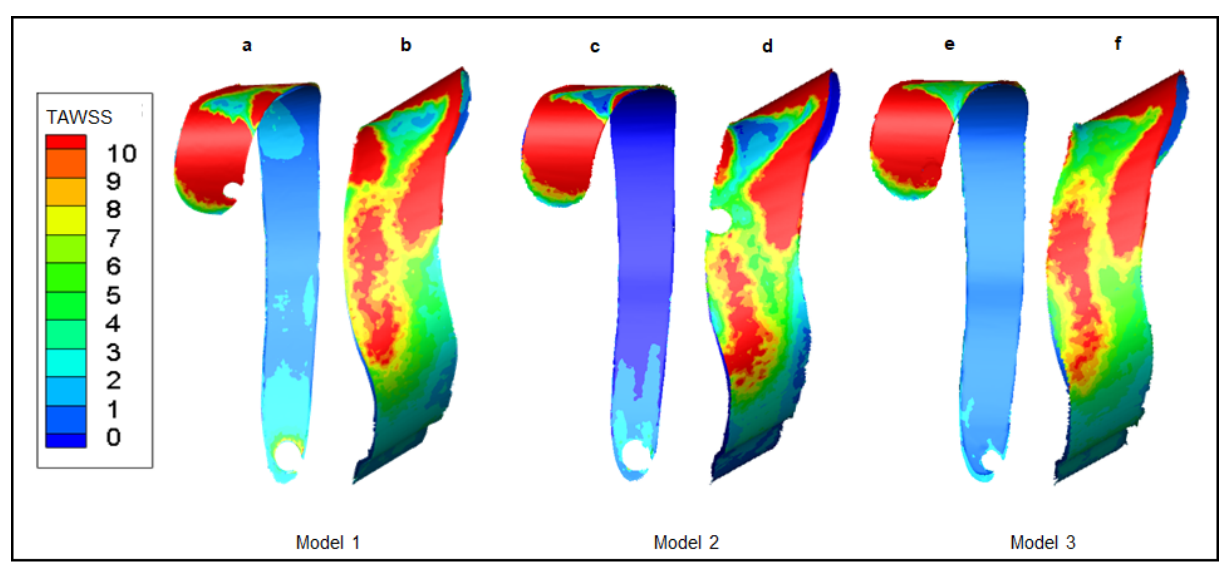

Figure 8: The TAWSS of three models. For a and $b$ illustrate the distribution of TAWSS in FL and TL of Model 1. For $\mathrm{c}$ and d show the distribution of TAWSS in FL and TL of Model 2. For e and f illustrate the distribution of TAWSS in FL and TL of Model 3 


\subsection{The distribution of RRT in the aorta}

Fig. 9 shows the distribution of RRT in the aorta. Figs. 9(a) and 9(b) illustrate the RRT distribution of Model 1. Figs. 9(c) and 9(d) show the RRT distribution of Model 2. Figs. 9(e) and 9(f) illustrate the RRT distribution of Model 3. As shown in Model 1, the region with a higher RRT was smaller and distributed almost in the aortic ligament of the FL. For Model 2 , the area with higher RRT was larger and distributed at ascending aorta entrance and descending aorta of FL. After covering the entry tear (Figs. 9(e), 9(f)), the distribution of RRT in the FL was extremely high, indicating that FL was almost thrombotic.

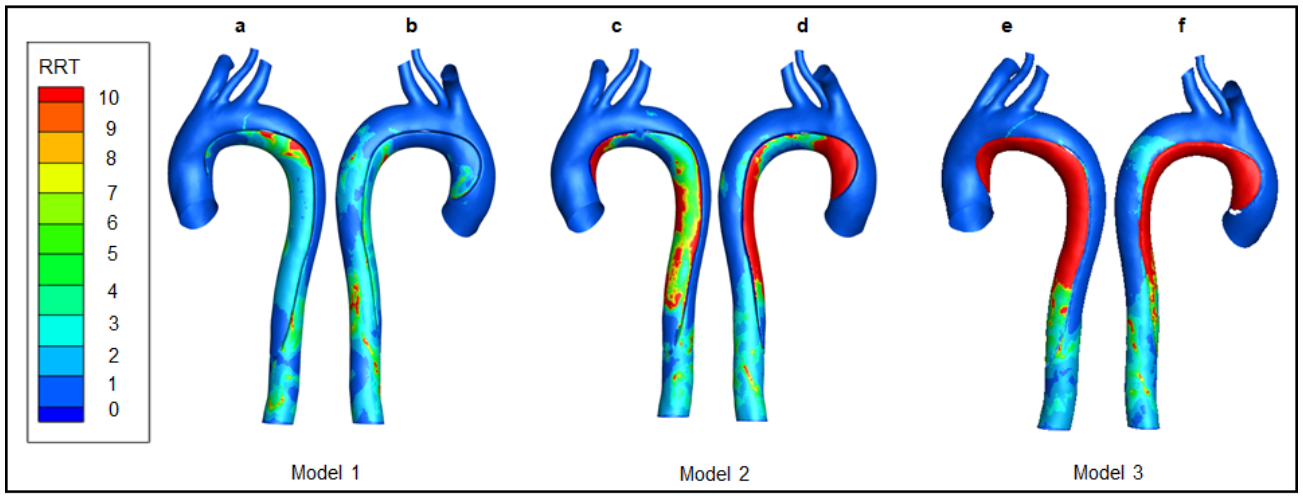

Figure 9: The distribution of RRT of three models. $(a, b)$ illustrate the RRT distribution of Model 1. (c, d) show the RRT distribution of Model 2. (e, f) illustrate the RRT distribution of Model 3

\section{Discussion}

At present, the evaluation of the pre-and post-treatment with type-A aortic dissection mainly relies on morphology imaging techniques. However, 4D PC-MRI cannot provide more details information about the pressure and flow pattern [Cheng, Juli, Wood et al. (2014)]. Previous studies have shown that the non-invasive method of CFD simulation can be used to investigate the changes of the pressure and flow pattern in the TL and FL of the aortic dissection [Gijsen, vandeVosse and Janssen (1999); Angouras, Sokolis, Dosios et al. (2000); Plonek, Zak, Rylski et al. (2018)]. CFD method can be used to predict the development of patients with aortic dissection and to make a surgical plan in time. This study emphasizes the changes in TL and FL hemodynamics of the type-A aortic dissection. Hemodynamic factors such as flow pattern, pressure, TAWSS and RRT, which are difficult to measure in vivo, can be defined through CFD simulation.

According to the results of the velocity field, we observed that the location of the entry tear has a significant effect on the hemodynamic changes in the TL and FL. The location of the entry tear is closer to the ascending aorta, resulting in a higher FL entry velocity and increased FL blood flow. Under this condition, the FL will continue to expand, increasing the risk of aortic rupture [Clough, Zymvragoudakis, Biasi et al. (2014)]. What's more, the vortex flow is found at the aortic arch of three models. The flow profiles in the aortic arch are generally skewed towards the inner curvature wall, which 
leads to the formation of the low-velocity zone in which secondary flow occurs. Mohiaddin R H et al. also found similar vortex flow in MRI studies [Mohiaddin, Bogren, Yang et al. (1994)]. Disordered aortic arch blood flow can easily lead to the occurrence of aortic aneurysm and aortic dissection tear [Malek, Alper and Izumo (1999); Slager, Wentzel, Gijsen et al. (2005)]. Simon et al. [Simon, Liu, Wong et al. (2016)] suggested that reducing the flow rate in FL by covering the entrance tear, and that blood flow would be more regular. This conclusion is in agreement with the results of this study.

From the analysis of the pressure, the area of the high-pressure difference between the TL and FL of each model is unique and related to structural changes (different position of the entry tear). The pressure of the FL is higher than TL (Model 1, the entry tear was located at the entrance of the ascending aorta), which may cause the oscillation of the dissection diaphragm, and may also cause the FL to continue to expand and compress the TL, ultimately leading to the occlusion of the TL. The surgical treatment should be given priority. After TEVAR (Model 3), the FL is compressed and TL is dilated. Therefore, the blood hemodynamic in the TL is completely different compared to the case before the surgical procedure [Polanczyk, Piechota-Polanczyk, Domenig et al. (2018)]. Reducing the pressure in the FL by covering the entry tear may be helpful in the treatment of early retrograde type-A dissection [Karmonik, Bismuth, Shah et al. (2011)].

The WSS plays an important role in the function of vascular endothelial cell proliferation, apoptosis, migration, and permeability [Fillinger, Raghavan, Marra et al. (2002); Prokop, Palmer and Wheat (1970)]. Xu et al. [Xu, Borghi, Nchimi et al. (2010)] also emphasized that early signs of ruptured abdominal aortic aneurysm occurred at the relatively high wall stress and fluid wall shear stress. According to the results of the TAWSS in this study, high TAWSS (TL TAWSS $>8 \mathrm{~Pa}, \mathrm{FL}$ TAWSS $=3 \mathrm{~Pa}$ ) was observed at the aortic ligament, which may be closely related to the complex vortex here. In Model 1, combined with the entry and re-entry tear, it can be easily inferred that high TAWSS leads to an expansion of the tear, which ultimately leads to aortic rupture [Tse, Chiu, Lee et al. (2011)]. After TEVAR, the high WSS area at the re-entry tear of descending aorta was reduced. Compared with pre-TEVAR, Polanczyk et al. also significantly reduced the WSS value of the aorta after TEVAR [Polanczyk, Piechota-Polanczyk, Neumayer et al. (2019)].

The RRT is a very important hemodynamic factor reflecting the effect of solute and blood residence time on fibrin polymerization, thrombus formation, and vascular permeability in blood vessels [Himburg, Grzybowski, Hazel et al. (2004)]. The blood enters into the FL through the entry tear and enters out of the FL through the re-entry tear, which reduces the formation of the FL thrombus [Tse, Chiu, Lee et al. (2011)]. Covering the entry tear, the pressure of the FL was reduced and almost complete thrombotic. The FL patency is considered to be the main predictor of aneurysm expansion, and complete thrombosis has been clinically proven to improve the prognosis of the disease [Chen, Müller-Eschner, Kotelis et al. (2013)]. Therefore, the position of the tear has an important effect on FL thrombosis. This work was used as a preliminary study to demonstrate the potential applicability of our study to predict thrombosis after TEVAR.

The morphological and hemodynamic factors of the artery are complementary. Arterial geometry plays a key role in the nature of hemodynamic models, which in turn affects the 
arterial geometry. Although the method of this study does not consider the mechanical properties of vascular tissue, it has been proved feasible to regard aortic dissection as a rigid structure when the composition of aortic dissection is inaccurate. This may be a preliminary study of the position of aortic dissection tear and the results of simulated TEVAR surgery [Shi, Zhu, Chang et al. (2016); Polanczyk, Podgorski, Polanczyk et al. (2018)].

Limitations of this study: Firstly, due to the complex geometry of the aortic dissection and the lack of real material properties, we assumed that the fluid boundary was a rigid structure, ignoring the effects of fluid deformation and vessel wall on the fluid. Secondly, due to the lack of patient-specific blood flow information, the boundary conditions, which have been validated by various in vivo experimental data [Fung (2013)], and have been widely used in various numerical simulations of healthy and unhealthy aortas [Cheng, Lam, Fung et al. (2008); Lam, Fung, Cheng et al. (2008)], are used in this study. Thirdly, there is only one single position of the tear in the current study. Finally, some of the results were studied at the velocity vector peak systole, which may not fully reflect the hemodynamic changes of the whole cardiac cycle. In the next work, to get a more comprehensive and accurate conclusion, fluid-structure interaction should be used to study the simulation. At the same time, we will further study the individual blood flow information of the subjects, increase the position and number of the tears, and obtain hemodynamic information of the whole cardiac cycle.

\section{Conclusion}

This study has the following conclusions. The location of the entry tear has a significant effect on the hemodynamic changes in the aortic dissection. The blood flow velocity at the entry tear is higher than that of the surrounding area. The location of the entry tear is closer to the ascending aorta, which resulted in the increase of blood flow in the FL. Moreover, the pressure of the FL is higher than that of the TL. The intimal oscillation of aortic dissection is more severe, and high WSS appears at the re-entry tear. After covering the entry, the blood flow of FL is almost zero and the pressure of the FL decreased. The FL was almost thrombosis after covering the entry tear.

CFD method is used to obtain multi-dimensional hemodynamic information such as velocity field, pressure, WSS and RRT, which helps clinicians better understand the development of type A aortic dissection and provide the theoretical basis for clinical treatment. However, due to the limitation of work, further research is needed.

Acknowledgement: This study was partly funded by the National Natural Science Foundation of China (Grant Nos. 11602007, 11572014, 11832003), and the Key research and development program (2017YFC0111104), and New Talent (015000514118002).

\section{References}

Angouras, D.; Sokolis, D. P.; Dosios, T.; Kostomitsopoulos, N.; Boudoulas, H. et al. (2000): Effect of impaired vasa vasorum flow on the structure and mechanics of the thoracic aorta: implications for the pathogenesis of aortic dissection. European Journal of Cardio-Thoracic Surgery, vol. 17, no. 4, pp. 468-473. 
Alimohammadi, M.; Sherwood, J. M.; Karimpour, M.; Agu, O.; Balabani, S. et al. (2015): Aortic dissection simulation models for clinical support: fluid-structure interaction vs. rigid wall models. Biomedical Engineering Online, vol. 14, no. 1, pp. 34.

Aenis, M.; Stancampiano, A. P.; Wakhloo, A. K.; Lieber, B. B. (1997): Modeling of flow in a straight stented and nonstented side wall aneurysm model. Journal of Biomechanical Engineering, vol. 119, no. 2, pp. 206-212.

Bonfanti, M.; Balabani, S.; Alimohammadi, M.; Agu, O.; Homer-Vanniasinkam, S. et al. (2018): A simplified method to account for wall motion in patient-specific blood flow simulations of aortic dissection: comparison with fluid-structure interaction. Medical Engineering and Physics, vol. 58, pp. 72-79.

Cebral, J. R.; Castro, M. A.; Burgess, J. E., Pergolizzi, R. S.; Sheridan, M. J. et al. (2005): Characterization of cerebral aneurysms for assessing risk of rupture by using patientspecific computational hemodynamics models. American Journal of Neuroradiology, vol. 26 , no. 10 , pp. $2550-2559$.

Cheng, S. W.; Lam, E. S.; Fung, G. S.; Ho, P.; Ting, A. C. et al. (2008): A computational fluid dynamic study of stent graft remodeling after endovascular repair of thoracic aortic dissections. Journal of vascular surgery, vol.48, no. 2, pp. 303-310.

Chen, D.; Müller-Eschner, M.; Kotelis, D.; Böckler, D.; Ventikos, Y. et al. (2013): A longitudinal study of Type-B aortic dissection and endovascular repair scenarios: computational analyses. Medical Engineering and Physics, vol. 35, no. 9, pp. 1321-1330.

Cheng, Z.; Juli, C.; Wood, N. B.; Gibbs, R. G. J.; Xu, X. Y. (2014): Predicting flow in aortic dissection: comparison of computational model with PC-MRI velocity measurements. Medical Engineering and Physics, vol. 36, no. 9, pp. 1176-1184.

Clough, R. E.; Zymvragoudakis, V. E.; Biasi, L.; Taylor, P. R. (2014): Usefulness of new imaging methods for assessment of type B aortic dissection. Annals of Cardiothoracic Surgery, vol. 3, no. 3, pp. 314.

Fung, Y. C. (2013): Biomechanics: Circulation. Springer Science \& Business Media.

Fillinger, M. F.; Raghavan, M. L.; Marra, S. P.; Cronenwett, J. L.; Kennedy, F. E. (2002): In vivo analysis of mechanical wall stress and abdominal aortic aneurysm rupture risk. Journal of Vascular Surgery, vol. 36, no. 3, pp. 589-597.

Golledge, J.; Eagle, K. A. (2008): Acute aortic dissection. Lancet, vol. 372, no. 9632, pp. 55-66.

Gijsen, F. J.; vandeVosse, F. N.; Janssen, J. D. (1999): The influence of the nonNewtonian properties of blood on the flow in large arteries: steady flow in a carotid bifurcation model. Journal of Biomechanics, vol. 32, no. 6, pp. 601-608.

Hanna, J. M.; Andersen, N. D.; Ganapathi, A. M.; McCann, R. L.; Hughes, G. C. (2014): Five-year results for endovascular repair of acute complicated type B aortic dissection. Journal of Vascular Surgery, vol. 59, no. 1, pp. 96-106.

Himburg, H. A.; Grzybowski, D. M.; Hazel, A. L.; LaMack, J. A.; Li, X. M. et al. (2004): Spatial comparison between wall shear stress measures and porcine arterial endothelial permeability. American Journal of Physiology-Heart and Circulatory Physiology, vol. 286, no. 5, pp. H1916-H1922. 
Hagan, P. G.; Nienaber, C. A.; Isselbacher, E. M.; Bruckman, D.; Karavite, D. J. et al. (2000): The International registry of acute aortic dissection (IRAD): new insights into an old disease. Journal of the American Medical Association, vol. 283, no. 7, pp. 897-903.

Hebballi, R.; Swanevelder, J. (2009): Diagnosis and management of aortic dissection. Continuing Education in Anaesthesia, Critical Care and Pain, vol. 9, no. 1, pp. 14-18.

Ku, D. N. (1997). Blood flow in arteries. Annual Review of Fluid Mechanics, vol. 29, no. 1, pp. 399-434.

Karmonik, C.; Bismuth, J.; Shah, D. J.; Davies, M. G.; Purdy, D. et al. (2011): Computational study of haemodynamic effects of entry-and exit-tear coverage in a DeBakey type III aortic dissection: technical report. European Journal of Vascular and Endovascular Surgery, vol. 42, no. 2, pp. 172-177.

Kabinejadian, F.; Chua, L. P.; Ghista, D. N.; Sankaranarayanan, M.; Tan, Y. S. (2010): A novel coronary artery bypass graft design of sequential anastomoses. Annals of Biomedical Engineering, vol. 38, no. 10, pp. 3135-3150.

Kallenbach, K.; Oelze, T.; Salcher, R. (2005): Evolving strategies for treatment of acute aortic dissection type A. ACC Current Journal Review, vol. 1, no. 14, pp. 59.

Lam, S. K.; Fung, G. S.; Cheng, S. W.; Chow, K. W. (2008): A computational study on the biomechanical factors related to stent-graft models in the thoracic aorta. Medical \& Biological Engineering \& Computing, vol. 46, no. 11, pp. 1129-1138.

Malek, A. M.; Alper, S. L.; Izumo, S. (1999): Hemodynamic shear stress and its role in atherosclerosis. Journal of the American Medical Association, vol. 282, no. 21, pp. 2035-2042.

Mohiaddin, R. H.; Bogren, H. G.; Yang, G. Z.; Kilner, P. J.; Firmin, D. N. (1994): Magnetic resonance velocity vector mapping in aortic aneurysms. Magnetic Resonance Materials in Physics, Biology and Medicine, vol. 2, no. 3, pp. 335-338.

Morris, L.; Delassus, P.; Walsh, M.; McGloughlin, T. (2004): A mathematical model to predict the in vivo pulsatile drag forces acting on bifurcated stent grafts used in endovascular treatment of abdominal aortic aneurysms (AAA). Journal of Biomechanics, vol. 37, no. 7, pp. 1087-1095.

Macura, K. J.; Corl, F. M.; Fishman, E. K.; Bluemke, D. A. (2003): Pathogenesis in acute aortic syndromes: aortic dissection, intramural hematoma, and penetrating atherosclerotic aortic ulcer. American Journal of Roentgenology, vol. 181, no. 2, pp. 309-316.

Olufsen, M. S.; Peskin, C. S.; Kim, W. Y.; Pedersen, E. M.; Nadim, A. et al. (2000): Numerical simulation and experimental validation of blood flow in arteries with structured-tree outflow conditions. Annals of Biomedical Engineering, vol. 28, no. 11, pp. 1281-1299.

Polanczyk, A.; Piechota-Polanczyk, A.; Domenig, C.; Nanobachvili, J.; Huk, I. et al. (2018): Computational fluid dynamic accuracy in mimicking changes in blood hemodynamics in patients with acute type IIIb aortic dissection treated with TEVAR. Applied Sciences, vol. 8, no. 8, pp. 1309. 
Polanczyk, A.; Piechota-Polanczyk, A.; Neumayer, C.; Huk, I. (2019): CFD reconstruction of blood hemodynamic based on a self-made algorithm in patients with acute type IIIb aortic dissection treated with TEVAR procedure. IUTAM Symposium on Recent Advances in Moving Boundary Problems in Mechanics, pp. 75-84.

Polanczyk, A.; Podgorski, M.; Polanczyk, M.; Veshkina, N.; Zbicinski, I. et al. (2018): A novel method for describing biomechanical properties of the aortic wall based on the three-dimensional fluid-structure interaction model. Interactive cardiovascular and thoracic surgery, vol.28, no. 2, pp. 306-315.

Prokop, E. K.; Palmer, R. F.; Wheat, M. W. (1970): Hydrodynamic forces in dissecting aneurysms: in-vitro studies in a tygon model and in dog aortas. Circulation Research, vol. 27, no. 1, pp. 121-127.

Plonek, T.; Zak, M.; Rylski, B.; Berezowski, M.; Czerny, M. et al. (2018): Wall stress correlates with intimal entry tear localization in Type A aortic dissection. Interactive Cardiovascular and Thoracic Surgery, vol. 27, no. 6, pp. 797-801.

Remke, K. (1981): The Fluid Mechanics of Large Blood Vessels, vol. 61, no. 3-5, pp. 207-207. Cambridge University Press, Cambridge.

Rapezzi, C.; Longhi, S.; Graziosi, M.; Biagini, E.; Terzi, F. et al. (2008): Risk factors for diagnostic delay in acute aortic dissection. American Journal of Cardiology, vol. 102, no. 10 , pp. 1399-1406.

Simon, C. H.; Liu, W.; Wong, R. H.; Underwood, M.; Wang, D. (2016): The potential of computational fluid dynamics simulation on serial monitoring of hemodynamic change in Type B aortic dissection. Cardiovascular and Interventional Radiology, vol. 39, no. 8, pp. 1090-1098.

Slager, C. J.; Wentzel, J. J.; Gijsen, F. J. H.; Thury, A.; Vander Wal, A. C. et al. (2005): The role of shear stress in the destabilization of vulnerable plaques and related therapeutic implications. Nature Reviews Cardiology, vol. 2, no. 9, pp. 456.

Shi, Y.; Zhu, M.; Chang, Y.; Qiao, H.; Liu, Y. (2016): The risk of stanford type-A aortic dissection with different tear size and location: a numerical study. Biomedical engineering online, vol. 15, no. 2, pp. 128.

Tse, K. M.; Chiu, P.; Lee, H. P.; Ho, P. (2011): Investigation of hemodynamics in the development of dissecting aneurysm within patient-specific dissecting aneurismal aortas using computational fluid dynamics (CFD) simulations. Journal of Biomechanics, vol. 44, no. 5 , pp. $827-836$.

Tsai, T. T.; Schlicht, M. S.; Khanafer, K.; Bull, J. L.; Valassis, D. T. et al. (2008): Tear size and location impacts false lumen pressure in an ex vivo model of chronic type B aortic dissection. Journal of Vascular Surgery, vol. 47, no. 4, pp. 844-851.

Wan Ab Naim, W. N.; Ganesan, P. B.; Sun, Z.; Lei, J.; Jansen, S. et al. (2018): Flow pattern analysis in type $\mathrm{B}$ aortic dissection patients after stent-grafting repair: Comparison between complete and incomplete false lumen thrombosis. International Journal for Numerical Methods in Biomedical Engineering, vol. 34, no. 5, e2961. 
Xu, X. Y.; Borghi, A.; Nchimi, A.; Leung, J.; Gomez, P. et al. (2010): High levels of 18 F-FDG uptake in aortic aneurysm wall are associated with high wall stress. European Journal of Vascular and Endovascular Surgery, vol. 39, no. 3, pp. 295-301.

Zhang, J.; Friedman, M. H. (2013). Adaptive response of vascular endothelial cells to an acute increase in shear stress frequency. American Journal of Physiology-Heart and Circulatory Physiology, vol. 305, no. 6, pp. H894-H902.

Zhao, X.; Liu, Y. J.; Bai, F.; Ren, X. C.; Ding, J. L. et al. (2012): Numerical study on bilateral bidirectional Glenn shunt. Journal of Medical Biomechanics, vol. 27, no. 5, pp. 488-494. 